\title{
A Kind of Unified Proper Efficiency in Vector Optimization
}

\author{
Ke Quan Zhao and Yuan Mei Xia \\ College of Mathematics Science, Chongqing Normal University, Chongqing 401331, China \\ Correspondence should be addressed to Ke Quan Zhao; kequanz@163.com
}

Received 22 February 2014; Accepted 14 April 2014; Published 30 April 2014

Academic Editor: Chong Li

Copyright (C) 2014 K. Q. Zhao and Y. M. Xia. This is an open access article distributed under the Creative Commons Attribution License, which permits unrestricted use, distribution, and reproduction in any medium, provided the original work is properly cited.

Based on the ideas of the classical Benson proper efficiency, a new kind of unified proper efficiency named S-Benson proper efficiency is introduced by using Assumption (B) proposed by Flores-Bazán and Hernández, which unifies some known exact and approximate proper efficiency including $(C, \varepsilon)$-proper efficiency and E-Benson proper efficiency in vector optimization. Furthermore, a characterization of $S$-Benson proper efficiency is established via a kind of nonlinear scalarization functions introduced by Göpfert et al.

\section{Introduction}

It is well known that approximate solutions have been playing an important role in vector optimization. Since Kutateladze initially introduced the concept of approximate solutions in [1], a lot of research achievements of approximate solutions have been obtained for vector optimization problems. Loridan proposed $\varepsilon$-efficient solutions of vector optimization problems and gave some properties in [2]. In a general topological vector space, Rong and $\mathrm{Wu}$ proposed $\mathcal{\varepsilon}$-weak efficient solutions of vector optimization problems with set-valued maps and obtained some linear scalarization theorems, Lagrangian multipliers theorems, saddle point theorems, and duality theorems in [3]. Recently, Gutiérrez et al. introduced the concept of coradiant set and proposed $(C, \varepsilon)$-efficient solutions which extend and unify some known different notions of approximate solutions in [4]. Gao et al. proposed the concept of properly approximate efficient solutions by means of coradiant set and established some linear and nonlinear scalarization results in [5]. Furthermore, Gutiérrez et al. obtained some characterizations of this kind of approximate solutions in terms of linear scalarization in [6].

Moreover, Debreu introduced the concept of free disposal sets to deal with mathematical economic problems in [7]. In a finite dimensional space, Chicco et al. introduced the concepts of improvement sets and E-efficient solutions and obtained some characterizations in [8]. Improvement sets are close to free disposal sets and can be applied to study vector optimization problems as an important tool. In particular, Zhao and Yang obtained a unified stability result with perturbations by means of improvement sets in [9]. Furthermore, Gutiérrez et al. generalized the concepts of improvement sets and E-efficient solutions to a general real locally convex Hausdorff topological vector space and studied some linear scalarization results in [10]. Zhao and Yang proposed $E$-weak efficient solutions of vector optimization problems with set-valued maps and established some linear scalarization theorems, Lagrange multiplier theorems, saddle point criteria, and duality in [11]. Zhao and Yang introduced the concept of $E$-Benson proper efficiency which unifies some proper efficiency and obtained some linear scalarization theorems and Lagrange multiplier theorems of this kind of proper efficiency in [12]. Flores-Bazán and Hernández proposed Assumption (B) and obtained some complete scalarizations of solution sets of a class of unified vector optimization problems via nonlinear scalarization in [13]. In addition, Flores-Bazán and Hernández obtained some optimality conditions of a class of unified vector optimization problems under Assumption (B) in [14].

Motivated by the works of $[4,5,12,13]$, we present a new kind of unified proper efficiency named $S$-Benson proper efficiency by using Assumption (B) proposed by Flores-Bazán and Hernández. This kind of proper efficiency 
unifies some known exact and approximate proper efficiency including $(C, \varepsilon)$-proper efficiency and $E$-Benson proper efficiency in vector optimization. Furthermore, we also give a characterization of $S$-Benson proper efficiency via nonlinear scalarization.

\section{Preliminaries}

Let $X$ be a linear space and $Y$ a real Hausdorff locally convex topological linear space. For a subset $A$ of $Y$, we denote the topological interior, the topological closure, the boundary, and the complement of $A$ by int $A, \operatorname{cl} A, \partial A$, and $Y \backslash A$, respectively. A set $A$ is solid if int $A \neq \emptyset$ and is proper if $A$ is nonempty and $A \neq Y$. The cone generated by $A$ is defined as

$$
\text { cone } A=\{\alpha a \mid \alpha \geq 0, a \in A\} .
$$

Let $Y^{*}$ denote the topological dual space of $Y$. The positive dual cone of a subset $A \subset Y$ is defined as

$$
A^{+}=\left\{y^{*} \in Y^{*} \mid\left\langle y^{*}, y\right\rangle \geq 0, \forall y \in A\right\} .
$$

Let $K$ be a closed convex pointed cone in $Y$ with nonempty topological interior. For any $x, y \in Y$, we define

$$
x \leq_{K} y \Longleftrightarrow y-x \in K \text {. }
$$

In this paper, we consider the following vector optimization problem:

$$
\begin{array}{cc}
\min & f(x) \\
\text { s.t. } & x \in D,
\end{array}
$$

where $f: X \rightarrow Y$ and $\emptyset \neq D \subset X$.

We say that $A$ is a coradiant set if $A$ satisfies $\alpha d \in A$ for every $d \in A, \alpha>1$. Let $C \subset Y$ be a proper solid coradiant set and define

$$
C(\varepsilon)=\varepsilon C, \quad \forall \varepsilon>0, \quad C(0)=\bigcup_{\varepsilon>0} C(\varepsilon) .
$$

Lemma 1 (see [5]). Let $C$ be a proper solid convex coradiant set. Then,

$$
\begin{aligned}
& \text { (i) } C(0)+C(\varepsilon) \subset C(\varepsilon), \forall \varepsilon \geq 0 \text {; } \\
& \text { (ii) } \operatorname{int}(\operatorname{cl} C(\varepsilon))=\operatorname{int} C(\varepsilon), \forall \varepsilon>0 .
\end{aligned}
$$

Definition 2 (see [5]). Let $\varepsilon \geq 0$. A feasible point $\bar{x} \in D$ is said to be a $(C, \varepsilon)$-proper efficient solution of (VP) if

$$
\text { cl cone }(f(D)+C(\varepsilon)-f(\bar{x})) \cap(-C(0)) \subset\{0\} .
$$

Definition 3 (see [10]). A nonempty set $E \subset Y$ is said to be an improvement set with respect to $K$ if $0 \notin E$ and $E+K=E$.

Lemma 4 (see [10]). Let $E \subset Y$ be a nonempty set. If $E$ is an improvement set with respect to $K$, then $E^{+} \subset K^{+}$. Additionally, if $E \subset K$, then $E^{+}=K^{+}$.

Definition 5 (see [12]). Let $E \subset Y$ be an improvement set with respect to $K$. A feasible point $\bar{x} \in D$ is said to be an $E$-Benson proper efficient solution of (VP) if

$$
\mathrm{cl}(\text { cone }(f(D)+E-f(\bar{x}))) \cap(-K)=\{0\} .
$$

Flores-Bazán and Hernández introduced Assumption B as follows.

Assumption $B$ (see [13]). Consider that $0 \neq q \in Y$ and $S \subset Y$ is a proper (not necessary closed) set such that $0 \in \partial S$ and $\mathrm{cl}(Y \backslash(-S))+\mathbb{R}_{++} q \subset \operatorname{int}(Y \backslash(-S))$.

Remark 6. From Assumption B, we have the equivalence

$$
\begin{aligned}
\operatorname{cl}(Y \backslash(-S))+\mathbb{R}_{++} q \subset \operatorname{int}(Y \backslash(-S)) \\
\Longleftrightarrow \mathrm{cl} S+\mathbb{R}_{++} q \subset \operatorname{int} S .
\end{aligned}
$$

Lemma 7 (see [15]). Let $S \subset Y$ be any nonempty subset. Then, $\operatorname{cl}($ cone $S)=\operatorname{cl}($ cone $(\mathrm{cl} S))$.

\section{A Kind of Unified Proper Efficiency}

In this section, we propose a kind of unified proper efficiency of (VP) by means of Assumption B by using the idea of the classical Benson proper efficiency and discuss some relations with other proper efficiency.

Definition 8. Let $q$ and $S$ satisfy Assumption B. One says that $\bar{x} \in D$ is a $S$-Benson proper efficient solution of (VP) if

$$
\operatorname{cl}(\operatorname{cone}(f(D)+S-f(\bar{x}))) \cap(-\operatorname{cl}(\operatorname{cone}(\operatorname{conv}(S))))=\{0\} .
$$

Denote by $\operatorname{PAE}(f, S)$ the set of $S$-Benson proper efficient solutions of (VP).

Example 9. Let $Y=\mathbb{R}^{2}, q=(0,1), f(x)=x, D=\left\{\left(x_{1}, x_{2}\right) \mid\right.$ $\left.x_{1} \leq 0, x_{2} \geq 0\right\}$, and

$$
\begin{aligned}
S= & \left\{\left(x_{1}, x_{2}\right) \mid x_{1}-x_{2} \leq 0, x_{1} \in \mathbb{R}, x_{2}>0\right\} \\
& \cup\left\{\left(x_{1}, x_{2}\right) \mid-1 \leq x_{1} \leq 0, x_{2}=0\right\} .
\end{aligned}
$$

Since

$$
\operatorname{cl} S+\mathbb{R}_{++} q=\left\{\left(x_{1}, x_{2}\right) \mid x_{1}-x_{2}<0, x_{1} \in \mathbb{R}, x_{2}>0\right\}=\operatorname{int} S,
$$

then, from Remark 6, it follows that $q$ and $S$ satisfy Assumption B. Let $\bar{x}=(0,0) \in D$. Since

$$
\begin{aligned}
\mathrm{cl} & (\operatorname{cone}(f(D)+S-f(\bar{x}))) \\
& =\mathrm{cl}(\operatorname{cone}(\operatorname{conv}(S))) \\
& =\left\{\left(x_{1}, x_{2}\right) \mid x_{1}-x_{2} \leq 0, x_{1} \in \mathbb{R}, x_{2} \geq 0\right\},
\end{aligned}
$$

then

$$
\operatorname{cl}(\operatorname{cone}(f(D)+S-f(\bar{x}))) \cap(-\operatorname{cl}(\operatorname{cone}(\operatorname{conv}(S))))=\{0\} .
$$

Therefore, $\bar{x} \in \operatorname{PAE}(f, S)$.

In the following, we discuss some relations between $S$ Benson proper efficiency and some other proper efficiency. 
Theorem 10. Let $K \subset Y$ be a pointed closed convex cone, $S=$ int $K$, and $q \in$ int $K$. Then, $S$-Benson proper efficiency reduces to the Benson proper efficiency.

Proof. Since $K$ is a convex cone, then we have int $K+K=$ int $K$ and hence, by $0 \notin S$, we can obtain that $S$ is an improvement set with respect to $K$. Then, it follows from Remark 3.2 in [12] that

$$
\mathrm{cl}(Y \backslash(-S))+\mathbb{R}_{++} q \subset \operatorname{int}(Y \backslash(-S)) .
$$

For $0 \in \partial S, q$ and $S$ satisfy Assumption B. Assume that $\bar{x}$ is a $S$-Benson proper efficient solution of (VP) and then, from Proposition 4.1 in [16], we have

$$
\begin{aligned}
\operatorname{cl} & (\operatorname{cone}(f(D)+K-f(\bar{x}))) \cap(-K) \\
& =\operatorname{cl}(\operatorname{cone}(f(D)+\operatorname{int} K-f(\bar{x}))) \cap(-K) \\
& =\operatorname{cl}(\operatorname{cone}(f(D)+S-f(\bar{x}))) \cap(-\operatorname{cl}(\operatorname{cone}(\operatorname{conv}(S)))) \\
& =\{0\},
\end{aligned}
$$

which implies that $\bar{x}$ is a Benson proper efficient solution of (VP).

Theorem 11. Let $K \subset Y$ be a pointed closed convex set and $q \in \operatorname{int} K$. If $S=E \subset K$ is an improvement set with respect to $K$ and $0 \in \partial S$, then $S$-Benson proper efficiency reduces to the E-Benson proper efficiency.

Proof. From Remark 3.2 in [12], we know that $q$ and $S$ satisfy Assumption B. Assume that $\bar{x}$ is $S$-Benson proper efficient solution of (VP). We first point out that

$$
\mathrm{cl}(\operatorname{cone}(\operatorname{conv} S))=K \text {. }
$$

In fact, since $S \subset K$, then we only need to prove

$$
K \subset \operatorname{cl}(\text { cone }(\operatorname{conv} S)) \text {. }
$$

Suppose that there exists $k_{0} \in K$ such that $k_{0} \notin$ $\mathrm{cl}($ cone $(\operatorname{conv} S))$. By applying separation theorem for convex sets, it follows that there exists $\lambda \in Y^{*} \backslash\left\{0_{Y^{*}}\right\}$ such that

$$
\left\langle\lambda, k_{0}\right\rangle>\langle\lambda, e\rangle, \quad \forall e \in \mathrm{cl}(\text { cone }(\text { conv } S)) .
$$

Let $e=0$; we have

$$
\left\langle\lambda, k_{0}\right\rangle>0
$$

Furthermore, we can show that $-\lambda \in(\operatorname{cl}(\operatorname{cone}(\operatorname{conv} S)))^{+}=$ $S^{+}$. Since $S$ is an improvement set with respect to $K$ and by Lemma 4 , we can obtain

$$
-\lambda \in E^{+}=K^{+},
$$

which implies $\left\langle\lambda, k_{0}\right\rangle \leq 0$. This contradicts (18) and then (15) holds. Hence,

$$
\begin{gathered}
\operatorname{cl}(\text { cone }(f(D)+E-f(\bar{x}))) \cap(-K) \\
=\operatorname{cl}(\operatorname{cone}(f(D)+S-f(\bar{x}))) \\
\cap(-\operatorname{cl}(\text { cone }(\text { conv } S)))=\{0\} .
\end{gathered}
$$

This means that $\bar{x}$ is an $E$-Benson proper efficient solution of (VP).

Theorem 12. Let $C$ be a proper solid convex coradiant set, $q \in$ int $C(0), \varepsilon \geq 0, S=C(\varepsilon)$, and $0 \in \partial S$. Then, $S$-Benson proper efficiency reduces to $(C, \varepsilon)$-proper efficiency.

Proof. From the convexity of $S$ and Lemma 1(i), we have

$$
\begin{aligned}
\operatorname{cl} S+\operatorname{cl} C(0) & =\operatorname{cl} C(\varepsilon)+\operatorname{cl} C(0) \subset \operatorname{cl}(C(\varepsilon)+C(0)) \subset \operatorname{cl} C(\varepsilon) \\
& =\operatorname{cl} S
\end{aligned}
$$

and so, from $0 \in \operatorname{cl} C(0)$, it follows that

$$
\mathrm{cl} S+\operatorname{cl} C(0)=\operatorname{cl} S .
$$

We first point out that $q$ and $S$ satisfy Assumption B. In fact, we only need to prove

$$
Y \backslash(-\operatorname{int} S)+\mathbb{R}_{++} q \subset Y \backslash(-\operatorname{cl} S) .
$$

For any $x \in Y \backslash(-\operatorname{int} S)+\mathbb{R}_{++} q$, we only need to prove $x \notin$ $-\operatorname{cl} S$. On the contrary, suppose that $-x \in \operatorname{cl} S$. Since $x \in Y \backslash$ $(-$ int $S)+\mathbb{R}_{++} q$, then there exist

$$
x_{1} \in Y \backslash(-\operatorname{int} S), \quad x_{2} \in \mathbb{R}_{++} q
$$

such that $x=x_{1}+x_{2}$; that is, $-x_{1}=-x+x_{2}$. Hence, from Lemma 1(ii) and (22), we have

$$
\begin{aligned}
-x_{1} & \in \operatorname{cl} S+\mathbb{R}_{++} q \subset \operatorname{cl} S+\operatorname{int} C(0) \\
& \subset \operatorname{int}(\operatorname{cl} S+C(0)) \subset \operatorname{int}(\operatorname{cl} S+\operatorname{cl} C(0)) \\
& =\operatorname{int}(\operatorname{cl} S)=\operatorname{int} S,
\end{aligned}
$$

which contradicts $x_{1} \in Y \backslash(-\operatorname{int} S)$ and so $q$ and $S$ satisfy Assumption B. Furthermore, from $S \subset C(0)$ and by means of (22), similar with the proof of (15), we have

$$
\mathrm{cl}(\operatorname{cone}(\operatorname{conv}(\operatorname{cl} S)))=\operatorname{cl} C(0) .
$$

From Lemma 7, it follows that

$$
\begin{aligned}
\mathrm{cl}(\text { cone }(\text { convS })) & =\mathrm{cl}(\text { cone } S)=\mathrm{cl}(\text { cone }(\operatorname{cl} S)) \\
& =\mathrm{cl}(\operatorname{cone}(\operatorname{conv}(\operatorname{cl} S)))=\operatorname{cl} C(0) .
\end{aligned}
$$

If $\bar{x}$ is $S$-Benson proper efficient solution of (VP), then

$$
\begin{aligned}
& \operatorname{cl}(\text { cone }(f(D)+C(\varepsilon)-f(\bar{x}))) \cap(-\operatorname{cl} C(0)) \\
& \quad=\operatorname{cl}(\operatorname{cone}(f(D)+S-f(\bar{x}))) \cap(-\operatorname{cl}(\text { cone }(\text { convS }))) \\
& \quad=\{0\}
\end{aligned}
$$

It follows that

$$
\mathrm{cl}(\text { cone }(f(D)+C(\varepsilon)-f(\bar{x}))) \cap(-C(0)) \subset\{0\},
$$

which implies that $\bar{x}$ is a $(C, \varepsilon)$-proper efficient solution of (VP). 


\section{A Characterization via Nonlinear Scalarization}

In this section, we give a characterization of $S$-Benson proper efficiency of (VP) via a kind of nonlinear scalarization function proposed by Göpfert et al.

Definition 13. Let $\xi_{q, S}: Y \rightarrow \mathbb{R} \cup\{ \pm \infty\}$ be defined by

$$
\xi_{q, S}(y)=\inf \{t \in \mathbb{R} \mid y \in t q-S\}, \quad y \in Y,
$$

with $\inf \emptyset=+\infty$.

Flores-Bazán and Hernández obtained the following nonconvex separation theorem.

Lemma 14 (see [13]). Let $q$ and S satisfy Assumption B. Then,

$$
\begin{gathered}
\left\{y \in Y \mid \xi_{q, S}(y)<c\right\}=c q-\operatorname{int} S, \quad \forall c \in \mathbb{R}, \\
\left\{y \in Y \mid \xi_{q, S}(y)=c\right\}=c q-\partial S, \quad \forall c \in \mathbb{R}, \\
\xi_{q, S}(-S) \leq 0, \quad \xi_{q, S}(-\partial S)=0 .
\end{gathered}
$$

We consider the following scalar optimization problem

$$
\left(P_{q, y}\right) \min _{x \in D} \xi_{q, S}(f(x)-y),
$$

where $y \in Y$ and $q \in Y$. Denote $\xi_{q, S}(f(x)-y)$ by $\left(\xi_{q, S, y} \circ f\right)(x)$ Let $\epsilon \geq 0$ and $\bar{x} \in D$. If

$$
\left(\xi_{q, S, y} \circ f\right)(x) \geq\left(\xi_{q, S, y} \circ f\right)(\bar{x})-\epsilon, \quad \forall x \in D,
$$

then $\bar{x}$ is called an $\epsilon$-minimal solution of $\left(P_{q, y}\right)$. Denote the set of $\epsilon$-minimal solutions of $\left(P_{q, y}\right)$ by $\operatorname{AMin}\left(\xi_{q, S, y} \circ f, \epsilon\right)$.

Theorem 15. Let $q \in \operatorname{int} S$ and $S$ satisfy Assumption $B$ and $\beta=\inf \left\{t \in \mathbb{R}_{+} \mid t q \in S\right\}$. Then,

$$
\bar{x} \in \operatorname{PAE}(f, S) \Longrightarrow \bar{x} \in \operatorname{AMin}\left(\xi_{q, S, f(\bar{x})} \circ f, \beta\right) .
$$

Proof. Since $\bar{x} \in \operatorname{PAE}(f, S)$, then

$$
\operatorname{cl}(\text { cone }(f(D)+S-f(\bar{x}))) \cap(-\operatorname{cl}(\text { cone }(\operatorname{conv} S)))=\{0\},
$$

and it follows that

$$
(f(D)+S-f(\bar{x})) \cap(-\operatorname{int}(\operatorname{cl}(\text { cone }(\text { convS }))))=\emptyset .
$$

Therefore,

$$
(f(\bar{x})-(S+\operatorname{int}(\operatorname{cl}(\text { cone }(\operatorname{conv} S))))) \cap f(D)=\emptyset .
$$

Furthermore, we can verify that

$$
\operatorname{int} S \subset S+\operatorname{int}(\operatorname{cl}(\text { cone }(\operatorname{conv} S))) \text {. }
$$

In fact, from Lemma 2.5 in [17], we have

$$
\begin{aligned}
\operatorname{int} S & \subset \operatorname{int}(S+\operatorname{cl}(\operatorname{cone}(\operatorname{conv} S))) \\
& \subset \operatorname{int}(\operatorname{cl} S+\operatorname{cl}(\operatorname{cone}(\operatorname{conv} S))) \\
& \subset \operatorname{int}(\operatorname{cl}(S+\operatorname{cone}(\operatorname{conv} S))) \\
& =S+\operatorname{int}(\operatorname{cone}(\operatorname{conv} S)) \\
& =S+\operatorname{int}(\operatorname{cl}(\operatorname{cone}(\operatorname{conv} S))) .
\end{aligned}
$$

Hence, from (37), we deduce that

$$
(f(D)-f(\bar{x})) \cap(-\operatorname{int} S)=\emptyset .
$$

From Lemma 14, we can obtain that, for all $c \in \mathbb{R}$,

$$
\left\{y \in Y \mid \xi_{q, S}(y)<c\right\}=c q-\operatorname{int} S .
$$

Let $c=0$ in (41); then we have

$$
\left\{y \in Y \mid \xi_{q, S}(y)<0\right\}=-\operatorname{int} S .
$$

It follows from (40) that

$$
(f(D)-f(\bar{x})) \cap\left\{y \in Y \mid \xi_{q, S}(y)<0\right\}=\emptyset .
$$

Therefore,

$$
\left(\xi_{q, S, f(\bar{x})} \circ f\right)(x)=\xi_{q, S}(f(x)-f(\bar{x})) \geq 0, \quad \forall x \in D .
$$

Now, we calculate $\left(\xi_{q, S, f(\bar{x})} \circ f\right)(\bar{x})$. In fact,

$$
\begin{aligned}
\left(\xi_{q, S, f(\bar{x})} \circ f\right)(\bar{x}) & =\xi_{q, S}(f(\bar{x})-f(\bar{x})) \\
& =\xi_{q, S}(0) \\
& =\inf \{t \in \mathbb{R} \mid 0 \in t q-S\} \\
& =\inf \{t \in \mathbb{R} \mid t q \in S\} \\
& \leq \inf \left\{t \in \mathbb{R}_{+} \mid t q \in S\right\}=\beta .
\end{aligned}
$$

Hence, from (44), we have

$$
\left(\xi_{q, S, f(\bar{x})} \circ f\right)(x) \geq\left(\xi_{q, S, f(\bar{x})} \circ f\right)(\bar{x})-\beta, \quad \forall x \in D,
$$

which means that

$$
\bar{x} \in \operatorname{AMin}\left(\xi_{q, S, f(\bar{x})} \circ f, \beta\right) .
$$

\section{Conflict of Interests}

The authors declare that there is no conflict of interests regarding the publication of this paper.

\section{Acknowledgments}

This work is partially supported by the National Natural Science Foundation of China (Grant nos. 11301574, 11271391, and 11171363), the Natural Science Foundation Project of Chongqing (Grant no. CSTC2012jjA00002), and the Research Fund for the Doctoral Program of Chongqing Normal University (13XLB029).

\section{References}

[1] S. S. Kutateladze, "Convex, $\varepsilon$-programming," Soviet Mathematics Doklady, vol. 20, no. 2, pp. 391-393, 1979. 
[2] P. Loridan, " $\varepsilon$-solutions in vector minimization problems," Journal of Optimization Theory and Applications, vol. 43, no. 2, pp. 265-276, 1984.

[3] W. D. Rong and Y. N. Wu, " $\varepsilon$-weak minimal solutions of vector optimization problems with set-valued maps," Journal of Optimization Theory and Applications, vol. 106, no. 3, pp. 569579, 2000.

[4] C. Gutiérrez, B. Jiménez, and V. Novo, "A unified approach and optimality conditions for approximate solutions of vector optimization problems," SIAM Journal on Optimization, vol. 17, no. 3, pp. 688-710, 2006.

[5] Y. Gao, X. Yang, and K. L. Teo, "Optimality conditions for approximate solutions of vector optimization problems," Journal of Industrial and Management Optimization, vol. 7, no. 2, pp. 483-496, 2011.

[6] C. Gutiérrez, L. Huerga, and V. Novo, "Scalarization and saddle points of approximate proper solutions in nearly subconvexlike vector optimization problems," Journal of Mathematical Analysis and Applications, vol. 389, no. 2, pp. 1046-1058, 2012.

[7] G. Debreu, Theory of Value, John Wiley, New York, NY, USA, 1959.

[8] M. Chicco, F. Mignanego, L. Pusillo, and S. Tijs, "Vector optimization problems via improvement sets," Journal of Optimization Theory and Applications, vol. 150, no. 3, pp. 516-529, 2011.

[9] K. Q. Zhao and X. M. Yang, "A unified stability result with perturbations in vector optimization," Optimization Letters, vol. 7, no. 8, pp. 1913-1919, 2013.

[10] C. Gutierrez, B. Jimenez, and V. Novo, "Improvement sets and vector optimization," European Journal of Operational Research, vol. 223, no. 2, pp. 304-311, 2012.

[11] K. Q. Zhao, X. M. Yang, and J. W. Peng, "Weak E-optimal solution in vector optimization," Taiwanese Journal of Mathematics, vol. 17, no. 4, pp. 1287-1302, 2013.

[12] K. Q. Zhao and X. M. Yang, "E-Benson proper efficiency in vector optimization," Optimization, 2013.

[13] F. Flores-Bazán and E. Hernández, "A unified vector optimization problem: complete scalarizations and applications," Optimization, vol. 60, no. 12, pp. 1399-1419, 2011.

[14] F. Flores-Bazán and E. Hernández, "Optimality conditions for a unified vector optimization problem with not necessarily preordering relations," Journal of Global Optimization, vol. 56, no. 2, pp. 299-315, 2013.

[15] X. M. Yang, D. Li, and S. Y. Wang, "Near-subconvexlikeness in vector optimization with set-valued functions," Journal of Optimization Theory and Applications, vol. 110, no. 2, pp. 413427, 2001.

[16] G. Y. Chen and W. D. Rong, "Characterizations of the Benson proper efficiency for nonconvex vector optimization," Journal of Optimization Theory and Applications, vol. 98, no. 2, pp. 365384, 1998.

[17] W. W. Breckner and G. Kassay, "A systematization of convexity concepts for sets and functions," Journal of Convex Analysis, vol. 4, no. 1, pp. 109-127, 1997. 


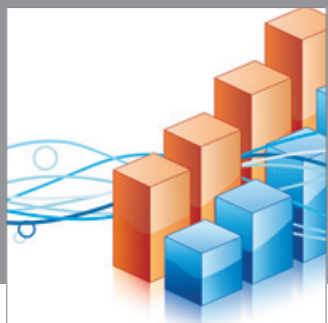

Advances in

Operations Research

mansans

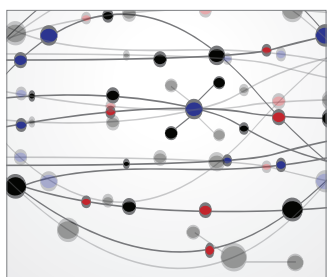

The Scientific World Journal
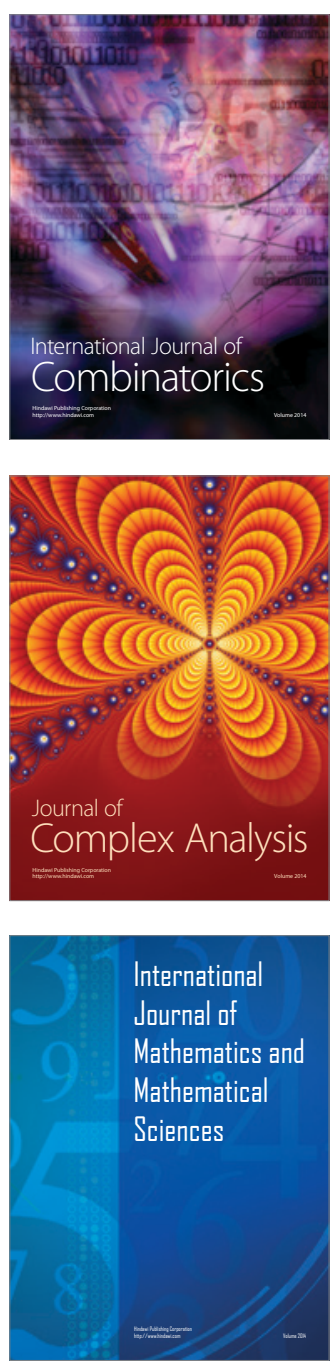
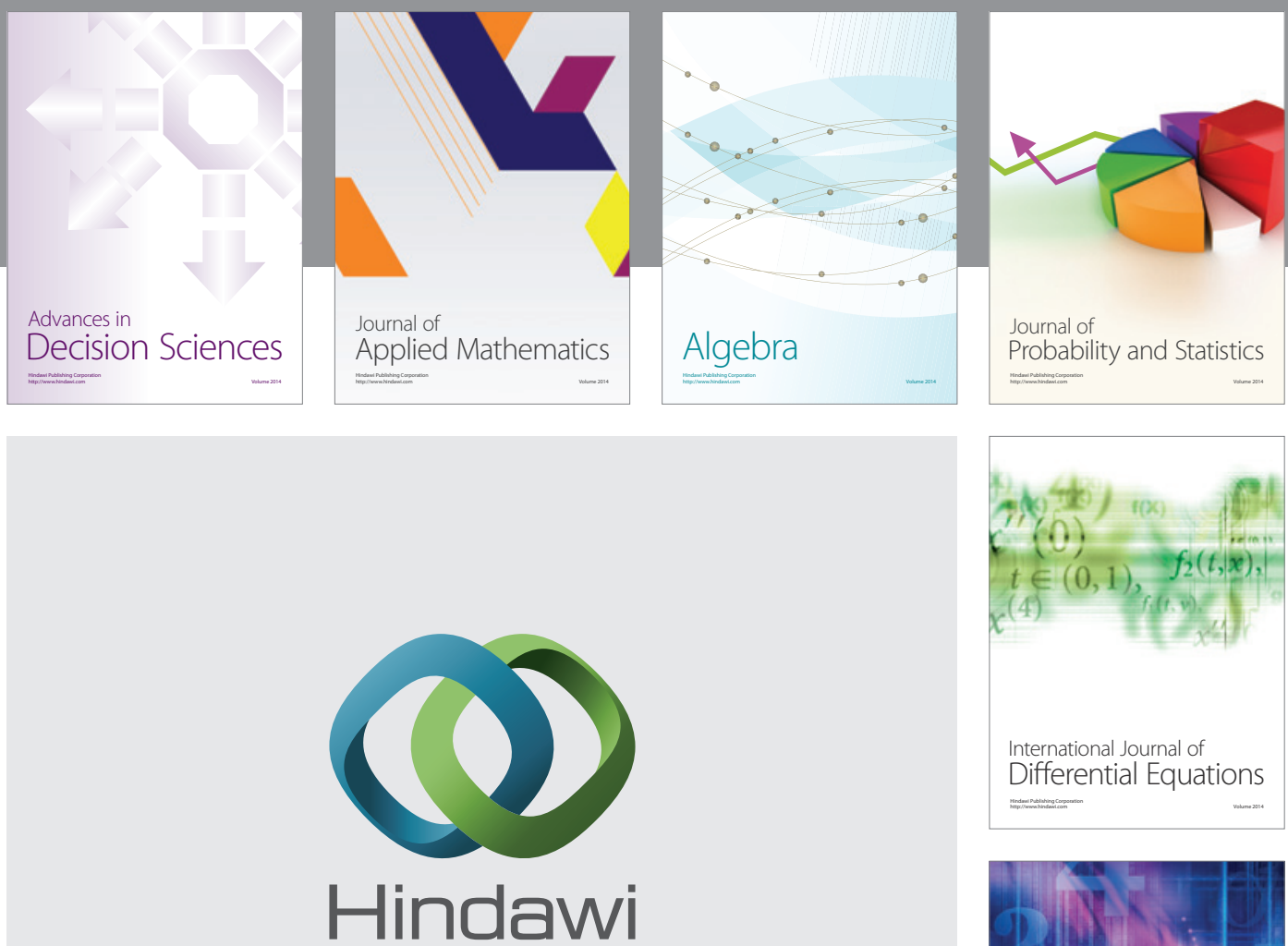

Submit your manuscripts at http://www.hindawi.com
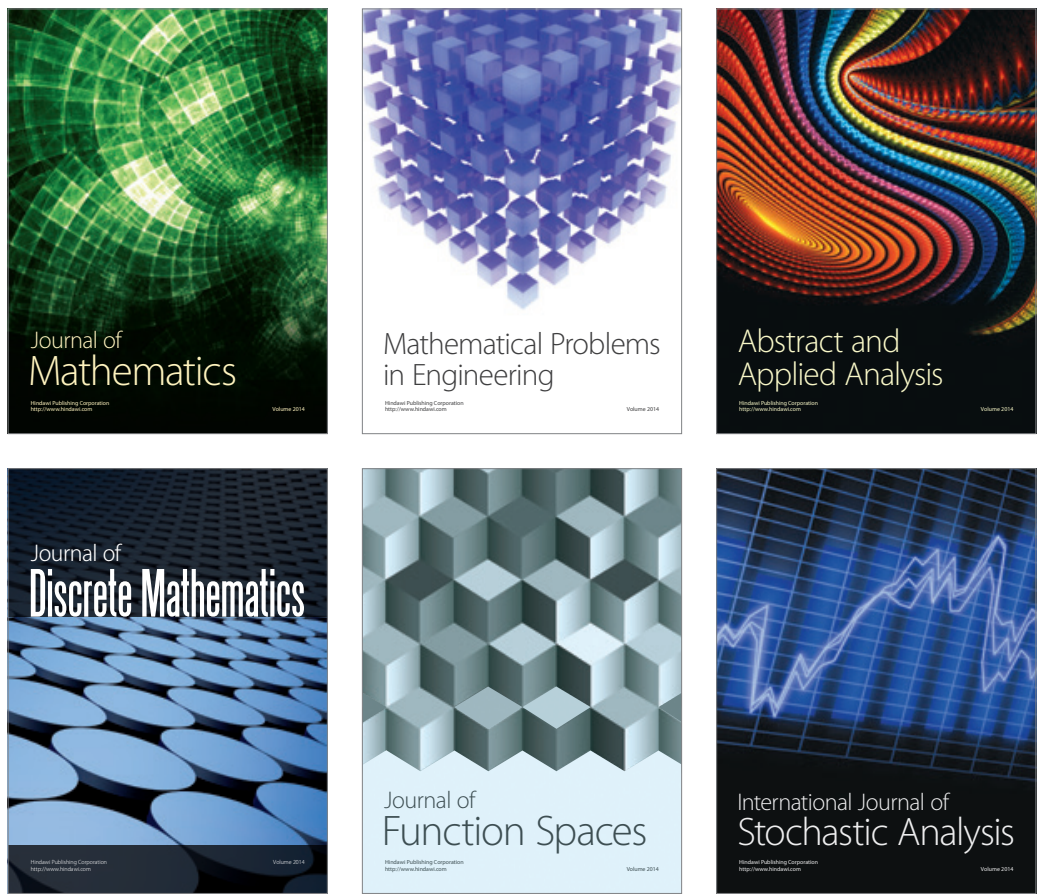

Journal of

Function Spaces

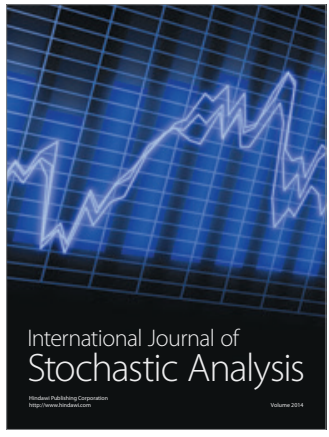

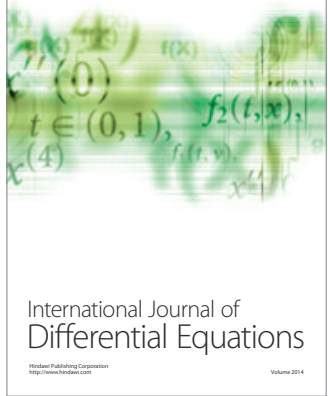
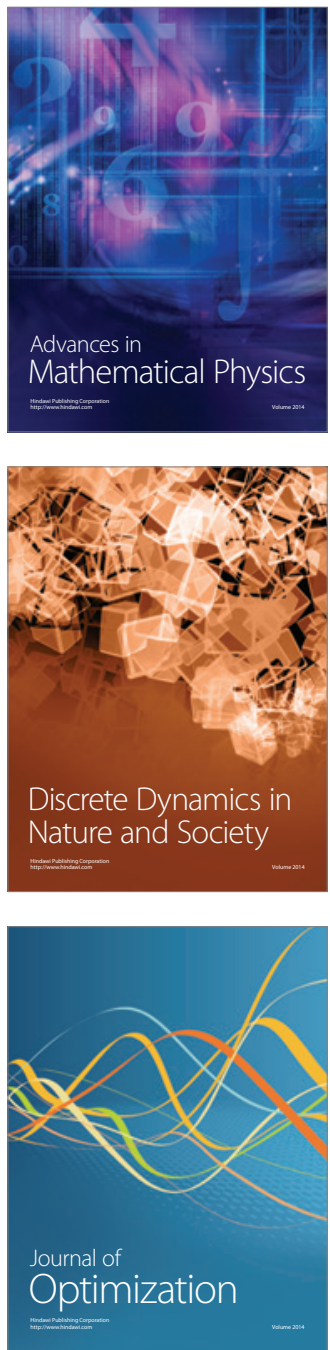